\title{
Transmandibular, circumglossal, retropharyngeal approach for chordomas of the clivus and upper cervical spine
}

\author{
Technical note
}

\author{
Franco DeMonte, M.D., F.R.C.S.(C), F.A.C.S., Eduardo Diaz, JR. M.D., \\ David Callender, M.D., M.B.A., AND IAN SuK, B.Sc., B.M.C. \\ Departments of Neurosurgery and Head and Neck Surgery, The University of Texas M. D. Anderson \\ Cancer Center, Houston, Texas
}

\begin{abstract}
Extensive clival tumors that involve both the midline and lateral skull base compartments, or those that extend inferiorly to the anterior cervical spine, are difficult to expose in a wide fashion using any of the transmaxillary, transoral, or transcervical routes. In the transmandibular, circumglossal, retropharyngeal (TCR) approach wide access of this region can be obtained, thus allowing for a more complete resection of tumor and infiltrated bone. It also provides for an improved ability to perform dural reconstruction, should it be necessary. Over the past 4 years four patients with extensive clival chordomas underwent resection via the TCR approach. Gross-total resection was achieved in two patients, a greater than $98 \%$ resection in one patient, and a greater than $95 \%$ resection in the fourth patient. The surgical technique, all approach-related complications and morbidity, and patient outcome are discussed. If an expanded exposure of the clivus is necessary, the TCR approach is a good choice as well as a useful surgical technique to have available.
\end{abstract}

\section{KEY WORDS • chordoma - transmandibular approach • clivus • cervical spine}

Chordomas are rare neoplasms accounting for 0.1 to $0.2 \%$ of all primary intracranial tumors. They are believed to originate from remnants of the primitive notochord and occur along the craniospinal axis. Approximately $35 \%$ of chordomas involve the cranium and two thirds of these lie in the clivus. The majority of clival chordomas are observed, at diagnosis, to be large and to extend to the anterior cranial fossa or parasellar region, whereas one third extend ventrally into the nasal cavities, the paranasal sinuses or the nasopharynx, or into the middle fossa. ${ }^{6}$ The lesions rarely affect only one anatomical region, thus complicating the choice of operative approach and decreasing the likelihood of complete resection.

Most often these tumors are soft and gelatinous in consistency but may be fibrous and tenacious, making removal difficult and dangerous, especially when using limited surgical approaches. Although they appear well localized, these tumors often involve and infiltrate surrounding bone. A wide surgical field allows more extensive bone removal with greater safety and facilitates dural repair, should it be necessary.

Abbreviations used in this paper: $\mathrm{CSF}=$ cerebrospinal fluid; $\mathrm{ICA}=$ internal carotid artery; MR = magnetic resonance; TCR = transmandibular, circumglossal, retropharyngeal.
Although Forsyth, et al., ${ }^{6}$ have reported a 5-year survival rate of nearly $50 \%$ and a 10 -year survival rate of only $30 \%$, a greater extent of resection was correlated with improved survival. Similarly, some authors ${ }^{7}$ who advocate radical resection of tumor and involved bone have reported survival rates of $65 \%$ at 5 years and diseasefree incidence rates of $70 \%$ at 2 years in their series. ${ }^{1}$ For these reasons, our philosophy has been to attempt maximum tumor resection in our patients harboring clival chordomas.

There have been numerous surgical approaches used to access the clivus. In choosing one approach over another, surgeons need to consider the parameters of tumor location, size, extension, unique patient anatomy, and functional requirements, as well as the experience, expertise, and preference of the surgical team. The anterior craniocervical junction is a difficult region to expose widely via any of the transmaxillary, transoral, or transcervical approaches. Large clival chordomas with anterior and inferior extensions can occupy this region, thus complicating their removal. The TCR approach, with a transpalatal extension if needed, can be used to obtain a wide exposure in this region, thus shortening and unifying the surgical field and facilitating aggressive removal of the tumor and infiltrated bone. Because of the inherent morbidity associated with this approach, it should be used judiciously in 


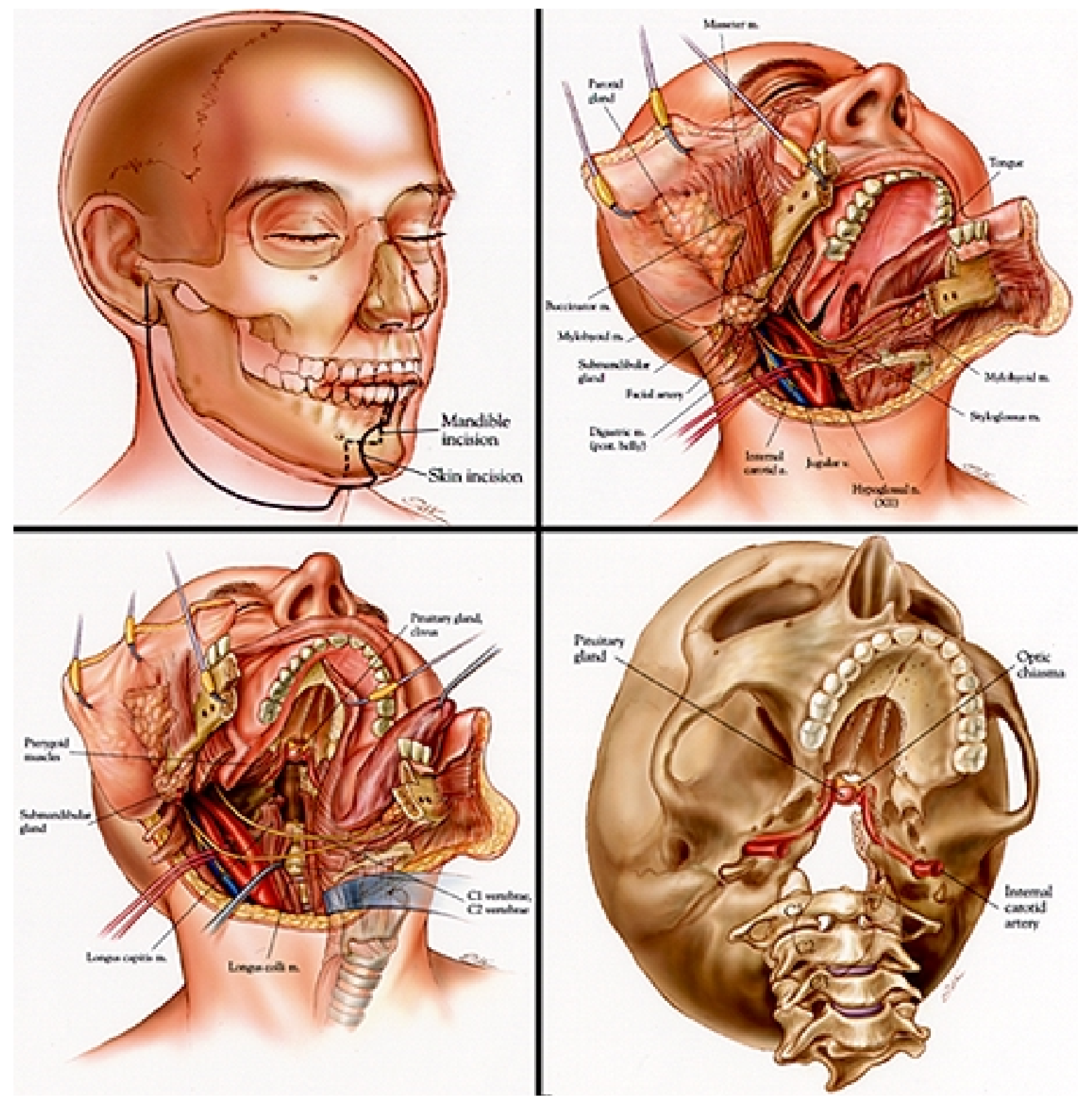

Fig. 1. Artist's drawings. Upper Left: The solid line depicts the skin incision and the dotted line the stairstep mandibular osteotomy necessary for the TCR approach. Upper Right: Following mandibulotomy and dissection of the upper neck the lingual and hypoglossal nerves, the ICA, external carotid artery, and internal jugular vein are exposed. The palatal incision is now underway. Note the predrilled holes in the mandible for placement of the reconstruction plate. Lower Left: The retractor is reflecting the pharynx. The longus colli muscles have been detached from the skull base on the patient's right side and are being dissected from the anterior spine. The clivus has been removed to expose the dura from the level of C-1 to the pituitary gland. Lower Right: Depiction of the osseous anatomy following removal of the clivus. Note the ICA is at risk both at the medial end of the formen lacerum and in the medial cavernous sinus. $a=$ artery; $\mathrm{m}=$ muscle; $\mathrm{n}=$ nerve; post $=$ posterior; $\mathrm{v}=$ vein .

cases in which surgical cure or significant palliation are likely.

\section{CLINICAL MATERIAL AND METHODS}

\section{Surgical Technique}

Following a tracheostomy, a curvilinear incision is be- gun just below the mastoid tip and extended inferiorly and medially along a skin crease to the mentum and through the lip (Fig. 1 upper left). Subplatysmal flaps are elevated to expose the upper neck, the submandibular gland, and surrounding tissues. A supraomohyoid neck dissection provides access to and clear identification of the lingual and hypoglossal nerves as well as the ICA and external 
Transmandibular, circumglossal, retropharyngeal approach

TABLE 1

Summary of data obtained in patients who underwent resection of chordoma via a TCR approach*

\begin{tabular}{|c|c|c|c|c|c|c|}
\hline $\begin{array}{l}\text { Case } \\
\text { No. }\end{array}$ & $\begin{array}{l}\text { Age (yrs), } \\
\text { Sex }\end{array}$ & $\begin{array}{c}\text { Presenting } \\
\text { Signs \& Symptoms }\end{array}$ & $\begin{array}{l}\text { Additional } \\
\text { Approach }\end{array}$ & $\begin{array}{l}\text { Extent of Resection/ } \\
\text { State of Dura }\end{array}$ & $\begin{array}{l}\text { Type of } \\
\text { Lesion }\end{array}$ & $\begin{array}{l}\text { Complications } \\
\text { of Approach }\end{array}$ \\
\hline 1 & $68, \mathrm{~F}$ & $\begin{array}{l}\text { serous otitis media, nasal obstruc- } \\
\text { tion, nasopharyngeal mass }\end{array}$ & transpalatal & GTR/intact & $\begin{array}{l}\text { chondroid } \\
\text { chordoma }\end{array}$ & $\begin{array}{l}\text { feeding tube for } 1 \mathrm{mo} \text {, } \\
\text { lingual neuropathy }\end{array}$ \\
\hline 2 & $29, \mathrm{~F}$ & headache & transcondylar & NTR/large defect & chordoma & none \\
\hline 3 & $19, \mathrm{M}$ & diplopia & transpalatal & GTR/intact & $\begin{array}{l}\text { chondroid } \\
\text { chordoma }\end{array}$ & $\begin{array}{l}\text { keloid, } 1.5-\mathrm{cm} \text { defect in } \\
\text { hard palate requiring } \\
\text { prosthesis }\end{array}$ \\
\hline 4 & $41, \mathrm{~F}$ & $\begin{array}{l}\text { nasopharyngeal numbness, odyno- } \\
\text { phagia, hoarseness, Lhermitte sign, } \\
\text { spasticity }\end{array}$ & transcondylar & STR/small defect & $\begin{array}{l}\text { chondroid } \\
\text { chordoma }\end{array}$ & $\begin{array}{l}\text { transient abducent paresis, } \\
\text { lingual neuropathy, soft- } \\
\text { palate fistula }\end{array}$ \\
\hline
\end{tabular}

* GTR = gross-total resection; NTR = near-total resection; STR = subtotal resection.

carotid artery and internal jugular vein. Continued elevation of the flap exposes the mandible. The mandibulotomy site is carefully marked, and an internal fixation plate is placed. The plate position is carefully marked and the plate removed and saved for closure. A stairstep mandibulotomy is performed between tooth 24 and 25 (the lowermiddle two incisors). Dissection follows the floor of the mouth posteriorly toward the glossopharyngeal sulcus, which allows the mandible to swing laterally and the tongue medially. The styloid process is palpated and the muscular attachments are separated. The ICA, internal jugular vein, and ninth, 10th, 11th, and 12th cranial nerves are traced superiorly to the skull base. As the incision approaches the anterior tonsillar pillar, it splits into two limbs. The upper limb of the incision extends to the soft palate, which is separated from its lateral attachments. This incision is then carried onto the hard palate approximately $1 \mathrm{~cm}$ medial to the alveolar ridge. It then passes anteriorly around to the contralateral hard palate. The greater palatine artery and nerve are transected, and the base of the hard palate is removed, as needed, for visualization into the sphenoid sinus. The lower limb of the incision extends into the hypopharynx, passing lateral to the tonsil and the orifice of the eustachian tube. The levator and tensor veli palatini muscles and the eustachian tube are transected, and retropharyngeal dissection is used to elevate the pharynx off of the longus colli muscles and expose the clivus and upper cervical spine. This pharyngeal flap is elevated and rotated medially (Fig. 1 upper right). The longus capiti muscles and the prevertebral fascia cover the clivus and upper cervical spine. Removal of the posterior hard palate reveals the posterior bony nasal septum and the anterior inferior face of the sphenoid sinus. Removal of this bone allows wide exposure of the sphenoid sinus and the sella turcica and upper clivus.

At this point, the tumor and clivus are resected (Fig. 1 lower left). Clival removal may extend from the sella turcica to the foramen magnum (Fig. 1 lower right). If the dura is deficient, repair with fascial and subcutaneous fat grafts and fibrin glue may be necessary.

Closure is begun by reattaching the superior constrictor muscle to the muscles at the base of the skull. The palatal flap is reapproximated, and the soft palate and hard palate mucosa is sutured. The preoperatively fashioned palatal stent is placed to support the palatal mucosa, and the floor of the mouth is closed in a double layer. The pharyngeal flap is allowed to return to its normal position and closure is performed by careful reapproximation of the posterolateral mucosal edges. The mandible is reapproximated and the reconstruction plate secured. Drains are placed in the neck, and a nasogastric tube is inserted. Precise alignment of the vermilion border is assured during lip closure, and the platysma and skin are closed in a standard fashion. Graduated, intermittent lumbar drainage is continued for 48 to 72 hours postoperatively if the dural closure is tenuous.

\section{Patient Data}

Between July 1996 and December 2000, four patients evaluated for clival chordoma were identified as being candidates for the TCR approach. There were three women and one man who ranged in age from 19 to 68 years (Table 1). In all four patients there was extensive disease with significant anterior and inferior extensions reaching the nasopharynx and upper cervical spine. In all cases surgery was performed via the TCR approach with or without palatectomy (Table 1). Two patients also required a transcondylar approach to maximize the tumor resection. Surgery-related complications are listed in Table 1. Although the dura was widely deficient in one patient and a small defect was present in another, CSF leakage did not occur. Dural repair was performed using fascia lata, subcutaneous fat, and fibrin glue. The repair was buttressed by replacement of the pharyngeal flap to its anatomical position. Lumbar fluid drainage was used in the two patients in whom dural repair was required. In all four patients it was necessary to place tympanostomy tubes on the side of the eustachian tube section. The lingual nerve was preserved in two patients and primarily repaired in two.

Based on operative inspection and postoperative serial MR imaging, a gross-total resection was achieved in two patients, a near-total resection $(>98 \%)$ in one patient, and a subtotal resection $(>95 \%)$ in one patient. Three of the chordomas were of the chondroid variety. Recurrence has already been noted in two of these three patients; grosstotal resection had been achieved in both (Table 2). Postrecurrence treatment of these two patients has consisted of proton beam irradiation (79.2-Gy and 75.6-Gy doses, respectively). The patient receiving the higher dose went on to develop bilateral radiation-induced optic neuropathy that progressed to blindness. At last follow-up 
TABLE 2

Summary of patient outcome data*

\begin{tabular}{|c|c|c|c|}
\hline $\begin{array}{c}\text { Case } \\
\text { No. }\end{array}$ & $\begin{array}{c}\text { Time to } \\
\text { Tumor } \\
\text { Recurrence }\end{array}$ & $\begin{array}{l}\text { Treatment at } \\
\text { Recurrence }\end{array}$ & Outcome \\
\hline 1 & $2 \mathrm{mos}$ & $\begin{array}{l}\text { proton beam } \\
\text { RT }(79.2 \mathrm{~Gy})\end{array}$ & dead of disease at $2.2 \mathrm{yrs}$ \\
\hline 2 & none at $3.3 \mathrm{yrs}$ & NA & alive $\mathrm{w} /$ disease at $3.3 \mathrm{yrs}$ \\
\hline 3 & $9 \operatorname{mos}$ & $\begin{array}{l}\text { proton beam } \\
\text { RT }(75.6 \mathrm{~Gy})\end{array}$ & alive $\mathrm{w} /$ disease at $1.3 \mathrm{yrs}$ \\
\hline 4 & none at $4 \mathrm{mos}$ & NA & alive $\mathrm{w} /$ disease at 4 mos \\
\hline
\end{tabular}

* NA = not applicable; $\mathrm{RT}=$ radiotherapy

evaluation three patients were alive with disease and one patient had died of the disease. In the patient who died there was evidence of distant metastases (Table 2).

\section{ILLUSTRATIVE CASE}

\section{Case 4}

This 41-year-old woman noted nasopharyngeal numbness, nasal obstruction, and odynophagia. During septoplasty and tonsillectomy a mass was noted in the tonsillar fossa. The lesion was diagnosed as chondroid chordoma based on examination of a biopsy specimen. The month prior to evaluation she noted the onset of hoarseness, and numbness and tingling in both hands and in the left C-2 dermatome. Lhermitte phenomenon was clearly described. Examination revealed hypertonicity and hyperreflexia of all four extremities. Imaging studies revealed a large clival tumor with extension to $\mathrm{C}-2$ (Fig. 2A). There was significant cervicomedullary compression. A left transcondylar approach was initially used to relieve the marked neural compression (Fig. 2B). A second-stage left-sided TCR approach was undertaken to remove the remainder of the tumor. A small dural opening was re- paired using subcutaneous fat and fibrin glue. Postoperatively the patient experienced transient abducent paresis and facial numbness secondary to sectioning of the lingual nerve. A small fistula of the soft palate is healing spontaneously. Postoperative MR imaging reveals a subtotal excision $(>95 \%)$ with stable residual tumor in the region of the left occipital condyle. (Fig. 2C)

\section{DISCUSSION}

Initially described by Biller, et al., ${ }^{4}$ and popularized first by Krespi and colleagues ${ }^{8-10}$ and later by Ammirati and associates, ${ }^{2,3}$ the TCR approach provides simultaneous exposure of the middle and lateral compartments of the skull base, allows excellent vascular control and access to the ninth through 12th cranial nerves, and by straight forward expansion allows an exposure that extends from the ipsilateral infratemporal fossa to the contralateral medial pterygoid plate; from the anterior cranial fossa to the lower clivus; and from the anterior cervical spine down to C-7 (Fig. 1 lower left). The main indications for this approach are 1) large tumors involving both the middle and lateral compartments and 2) tumors of the craniocervical junction and upper cervical spine. Ammirati and Bernardo ${ }^{2}$ have analytically evaluated the complex anterior approaches to the cranial base and identified the TCR approach as providing the greatest degree of exposure of both the midline and lateral compartments of the extracranial skull base. This approach was also shown to be best suited for exposure of the midline compartment of the intracranial skull base, but less so for the exposure of the lateral compartment of the intracranial skull base. Its limitation in this area is that access beyond the petrous ICA cannot be obtained. In fact, in our four patients it was difficult to visualize the ipsilateral horizontal portion of the ICA. The recurrent tumor identified in our first patient was in this area.

Although ideally suited for extradural disease, intradu-

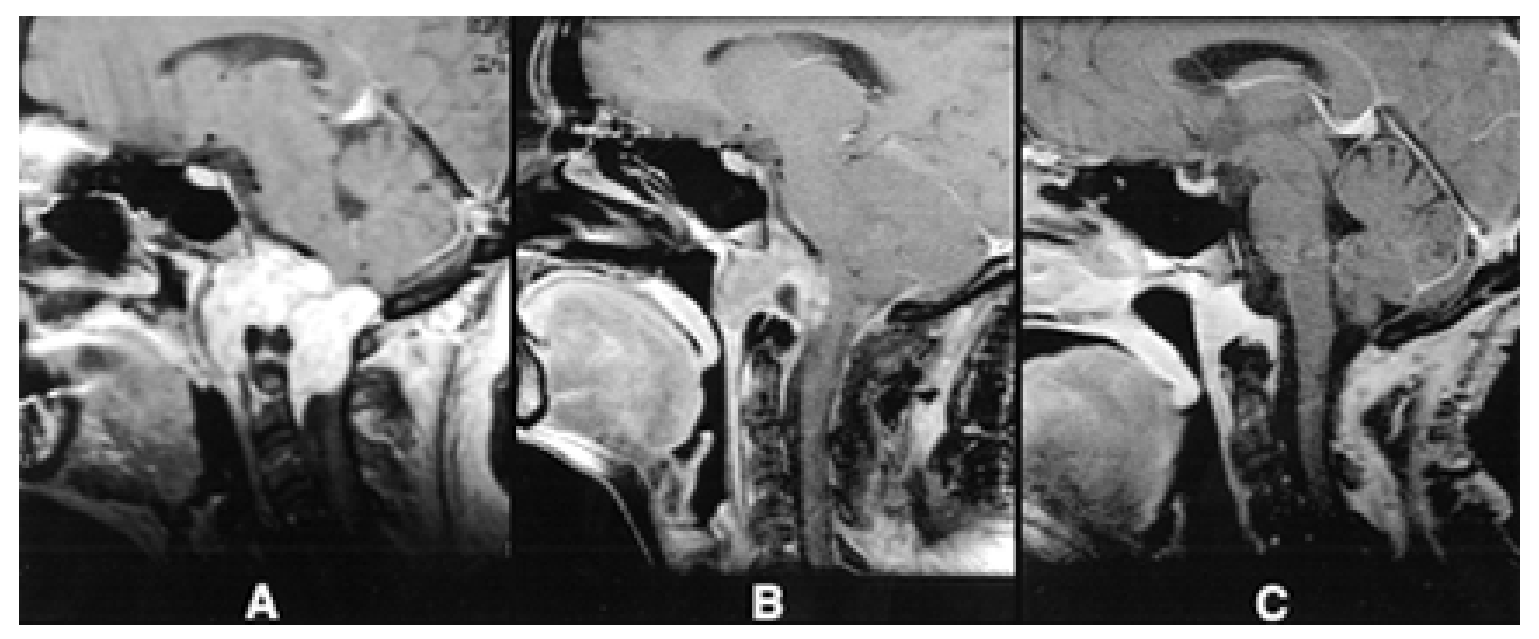

Fig. 2. Case 4. Sagittal $T_{1}$-weighted postcontrast MR images. A: Preoperative image. Note the anterior and inferior extension to the nasopharynx and upper cervical spine. The tumor is capping the C1-2 joint anteriorly. B: Image obtained following stage 1 transcondylar resection of the posterior component of the tumor. The cervicomedullary junction has been extensively decompressed. C: Image obtained following resection of residual tumor. Complete decompression of the cervicomedullary junction has been achieved. 


\section{Transmandibular, circumglossal, retropharyngeal approach}

ral extensions can certainly be removed by this approach; however, the risk of CSF leakage and meningitis is increased. Despite the fact that dural repair is rarely watertight in this region, the elevation and subsequent replacement of the laterally based pharyngeal flap helps to buttress any needed dural repairs. When combined with the judicious use of subcutaneous fat and fascial grafts, fibrin glue, and lumbar drainage, placement of the flap minimizes the incidence of CSF leakage. This is a distinctly different situation from that encountered when performing any of the anterior approaches that use a midline pharyngotomy. Unlike the TCR approach that raises a laterally based pharyngeal flap, which, when replaced, separates the surgical field from the aerodigestive tract, a midline pharyngotomy directly overlies the surgical field and places it in direct contact with the oropharynx. This is especially significant because the edges of a midline pharyngotomy can rarely, if ever, be completely approximated. Nagib, et al., ${ }^{11}$ reported a case of CSF leakage through a midline pharyngotomy that led to meningitis in a patient who had undergone resection of a clival chordoma. Although CSF leakage was not identified, Delagado, et al., ${ }^{5}$ have reported the occurrence of meningitis in one of their three patients following resection; a midline pharyngotomy was used. It seems, therefore, that of the complex anterior transfacial approaches to the clivus the TCR approach may be suited best for those chordomas that extend intradurally.

A good deal of anatomical dissection is required for this approach, which results in predictable morbidity. A temporary tracheostomy is required. Conductive hearing loss and serous otitis media result from sectioning of the eustachian tube. Temporary swallowing difficulties-induced by the circumglossal and palatal incisions, the extensive retropharyngeal dissection, and the sectioning of the tensor and levator veli palatini muscles-occasionally necessitate the insertion of a gastrostomy tube. The advantages of the TCR approach must, therefore, be carefully weighed against this increased morbidity. If exposure of the midline compartment of the extracranial skull base is all that is required - that is, without exposure of the region of the C1-2 joints - then approaches, such as the Lefort 1 approach, associated with a lower incidence of morbidity should be used. ${ }^{1,2}$ If expanded exposure of the midline skull base compartment is necessary, and especially if this needs to be combined with exposure of the lateral skull base compartment, the TCR approach is, in our opinion, the best choice.

\section{References}

1. Al-Mefty O, Borba LA: Skull base chordomas: a management challenge. J Neurosurg 86:182-189, 1997

2. Ammirati M, Bernardo A: Analytical evaluation of complex anterior approaches to the cranial base: an anatomic study. Neurosurgery 43:1398-1408, 1998

3. Ammirati M, Ma J, Cheatham ML, et al: The mandibular swing-transcervical approach to the skull base: anatomical study. Technical note. J Neurosurg 78:673-681, 1993

4. Biller HF, Shugar JM, Krespi YP: A new technique for widefield exposure of the base of the skull. Arch Otolaryngol 107: 698-702, 1981

5. Delgado TE, Garrido E, Harwick RD: Labiomandibular, transoral approach to chordomas in the clivus and upper cervical spine. Neurosurgery 8:675-679, 1981

6. Forsyth PA, Cascino TL, Shaw EG, et al: Intracranial chordomas: a clinicopathological and prognostic study of 51 cases. J Neurosurg 78:741-747, 1993

7. Gay E, Sekhar LN, Rubinstein E, et al: Chordomas and chondrosarcomas of the cranial base: results and follow-up of 60 patients. Neurosurgery 36:887-897, 1995

8. Krespi YP, Har-El G: Surgery of the clivus and anterior cervical spine. Arch Otolaryngol Head Neck Surg 114:73-78, 1988

9. Krespi YP, Sisson GA: Skull base surgery in composite resection. Arch Otolaryngol 108:681-684, 1982

10. Krespi YP, Sisson GA: Transmandibular exposure of the skull base. Am J Surg 148:534-538, 1984

11. Nagib MG, Wisiol ES, Simonton SC, et al: Transoral labiomandibular approach to basiocciput chordomas in childhood. Childs Nerv Syst 6:126-130, 1990

Manuscript received January 16, 2001.

Accepted in final form February 9, 2001.

Address reprint requests to: Franco DeMonte, M.D., F.R.C.S.(C) F.A.C.S., Department of Neurosurgery, Box 442, The University of Texas M. D. Anderson Cancer Center, 1515 Holcombe Boulevard, Houston, Texas 77030. email: fdemonte@mdanderson.org. 